\title{
Research and Application of Code Automatic Generation Algorithm Based on Structured Flowchart
}

\author{
Xiang-Hu Wu, Ming-Cheng Qu, Zhi-Qiang Liu, Jian-Zhong Li \\ School of Computer Science and Technology, Harbin Institute of Technology, Harbin, China. \\ Email: Wuxianghu@hit.edu.cn
}

Received June $17^{\text {th }}$, 2011; revised July $15^{\text {th }}, 2011$; accepted July $26^{\text {th }}, 2011$.

\begin{abstract}
It is of great significance to automatically generate code from structured flowchart. There are some deficiencies in existing researches, and their key algorithms and technologies are not elaborated, also there are very few full-featured integrated development platforms that can generate code automatically based on structured flowchart. By analyzing the characteristics of structured flowchart, a structure identification algorithm for structured flowchart is put forward. The correctness of algorithm is verified by enumeration iteration. Then taking the identified flowchart as input, an automatic code generation algorithm is proposed. Also the correctness is verified by enumeration iteration. Finally an integrated development platform is developed using those algorithms, including flowchart modeling, code automatic generation, $C D T \backslash G C C \mid G D B$ etc. The correctness and effectiveness of algorithms proposed are verified through practical operations.
\end{abstract}

Keywords: Automatic Generation of Codes, Structured Flowchart, Identification of Structure, Integrated Development Platform

\section{Introduction}

Software development ideas based on MDA (Model Driven Architecture) have attracted much attention from the research community in recent years [1,2]. MDA is first proposed by OMG. It is a methodology and standard system by which software systems are built on the basis of a variety of models, through model transformation to drive system development [3]. The development of Model-driven software is a hot issue in the current field of software engineering, and it has become a new software development paradigm to improve the quality and efficiency of software development [4]. Here the code generation indicates that a generator reads the code or documents related to the graphic model and generates high-level language program, just like $\mathrm{C}, \mathrm{C}++$, Java, Perl, Ruby, Python and HTML and so on..

There are many relevant researches about automatic code generation, such as: the solution, for automatic code generation based on metadata-driven, by the framework of .Net, achieve the objective of automatic generation of storage procedure and trigger in database [3]; A tool based on design pattern can automatically generate a design pattern of abstract level [4]; A automatic code generation technology based on uml meta model can generate the architecture of system, and meanwhile the generated code can reflect the hierarchy of original model [5];

By analyzing the synergy effect of the syntax environment between WWW and WEB, a WEB code automatic generation prototype system is proposed based on XML [6]. A UML tool can convert UML graphics into specific language to predict the performance of system [7]. In the field of multi-media, a data flow code automatic generation technology based on template can reduce the workload of direct memory access, meanwhile it can maintain the performance of multi-media computing [8].

A fast automatic code generator based on preferred pattern matching automata is proposed, it can be used to verify the correctness of UML state diagram and collaboration diagram, by insert primitives at fixed point of model, it can generate code automatically [9]. The tools, like I-Logix, Rhapsody, based on state machine, can generate code which can run at their real-time frameworks. 


\section{Related Research and Main Contribution}

\subsection{Related Research}

Flowchart plays an important role in system requirements analysis, preliminary design and detailed design aspects. It is particularly important when making communication and discussion, analysis and design of algorithms. But the traditional use of the flowchart are only limited to display, communication, description, and its role is only limited to graphical, intuitive, clear and easy communication and documentation compilation. So, automatic generation of a specific language code from flowchart will be a very important practical significance, it allows the designer to design the system from high-level functions without concerning for complicated code, and is more in line with the objective of MDA [10].

Recently, there are some reports about the automatic generation of code from flowchart. However, these researches all have certain deficiencies, and the core algorithm and technologies are not public, so the accuracy and validity are hard to be convinced. More researches, such as "AthTek Code to FlowChart", "Code to Chart", "AutoFlowchart" etc, are just its reverse engineering, that is automatic generation of flowchart from code,.

Hemlata Dakhore presented a strategy based on XML parser to generate code [11]. But the paper did not discuss how to identify the semantic of a specific flowchart. That is, the identification method of selection and loop are not discussed. According to the method, it must first determine whether a judgment node is a loop or selection, this information must be specified in advance by the modeler. If so it will lose the flexibility and convenience of a flowchart model, and also lack of automation and intelligence. And the paper only gives a sequence-selection simple example, for the algorithms of converting flowchart to XML and automatically generating code are not discussed. Martin C. Carlisle proposed a modeling and simulation system RAPTOR [12], which provides selection and loop primitives. This means that the modelers must know what kinds of structures they should draw in advance. While in standard flowchart there is only a judgment node, loop and selection nodes should be determined according to the semantic of a specific flowchart. So the RAPTOR is a specialized and nonstandard graphical language. And this article only describes the functions of a system. Tia Watts gave a flowchart modeling tool SFC, which can be used to automatically generate code [13]. But its operation is mechanical, can only inserted pre-standard graphical elements from fixed points, the flexibility is very low, operation is not convenient, lack of scalability, do not support the component model. Most importantly, it does not support nesting flowchart (processing nodes can be im- plemented as sub-flow chart). Kanis Charntaweekhun simply introduced the methods of how to use flow chart to program and its advantages, and said that the developed system can transform flowchart into code. But, the conversion algorithm, key technologies and data structures are not mentioned, and the examples given are very simple [14].

\subsection{Main Contributions}

Main contribution By analyzing the characteristics flowchart, we put forward a structure identification algorithm for structured flowchart, after then taking the flowchart identified in previous step as input, a algorithm which can generate code automatically is proposed. We verify the correctness and effectiveness of algorithms proposed using enumeration iteration strategy.

At last we designed and implemented an integrated development platform based on Eclipse and algorithms presented above; the platform uses a structured flowchart to describe program logic and can convert flowchart model into standard ANSI-C code. At the same time code editor (CDT), compilation tools (GCC) and debugging tools (GDB) are all integrated into this platform.

- Build the system with flowchart: Tasks and interrupt service can be modeled, platform can generate an instance of the task or interrupt, and also support nesting flowchart model and code generation.

- Variables and head-file management: Management of global variables, local variables, macros, and various header files.

\section{Structured Flowchart}

Any complex algorithms can be composed of three basic structures, sequence, selection and loop. These basic structures can be coordinates, they can include each other, but they can not cross and directly jump to another structure from the internal of a structure. As the whole algorithm is constructed by these three structures, just like composed by modules, therefore, it has the characteristics of clear structure, easily verifying accuracy and correcting errors $[15,16]$.

Flowchart is independent of any programming language. Structured flowchart can be further divided into five kinds of structures: sequence, selection, more selection, pre-check loop and post-check loop, as shown in Figure 1. Any complex flow chart can be built by the combination or the nest of the five basic control structures. Now there are many tools support flowchart modeling, such as Visio, Word, Rose and so on.

In order to make flowchart model more clear and intuitive and unambiguously, as shown in Figure 3, in addition to the order structure, the remaining four structures all use a judge node, when the executions exit their 


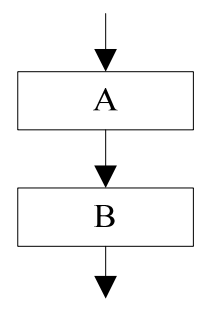

(1)sequence

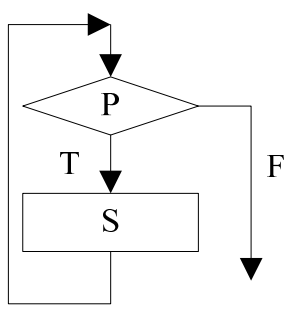

(3)while-do/for

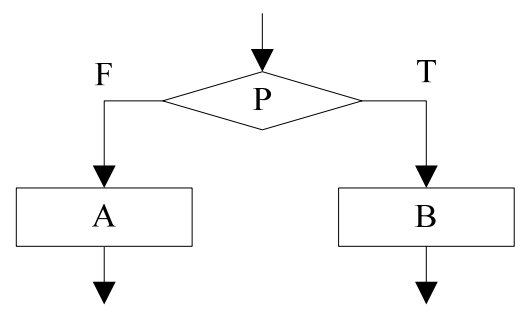

(2)if/if-else

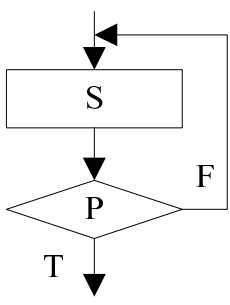

(4)do-while

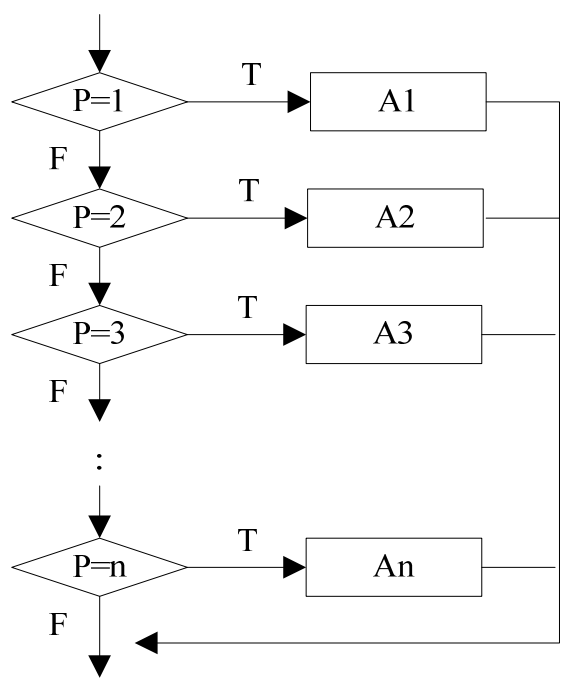

(5)case

Figure 1. Five structures of structured flowchart.
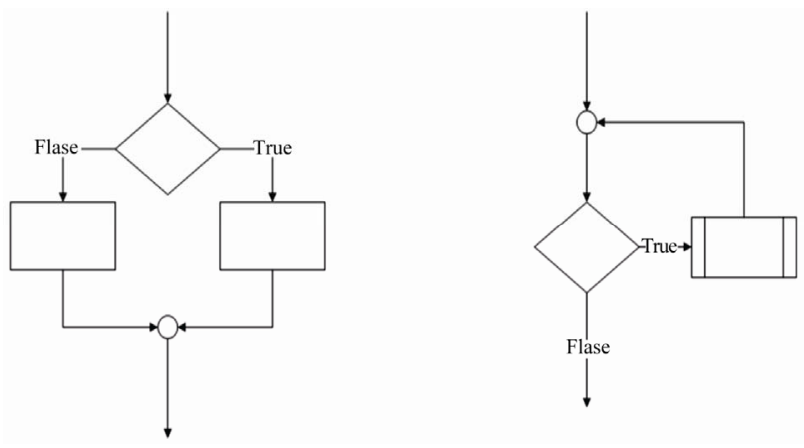

Figure 2. Examples of convergence.

structures, the page reference primitive ("o") must be used. It is called "on page reference" in visio, in this paper is called convergence, as shown in Figure 2.

In this paper we use the most commonly used five kinds of primitives for flowchart to automatic generating of code, and they are: "Begin”, "End", "Process", "Judgment" and "Convergence".

\section{Structure Identification}

\subsection{Identification Method}

\subsubsection{Identification of Basic Structure}

For the three basic structures shown in Figure 3 the loop structure must be a cycle path, while the sequence and selection structures must not be. Figure 3(a) and 3(b) both have a cycle path. For a basic structure, if a cycle path occurs in a Process node for the first time, its current father (comes from) must be a Judgment, if not, the flowchart must be wrong. We can identify the Judgment as a do-while structure. If a cycle path occurs in a Judgment node, we can also identify its current father (Judg- ment node) as a do-while structure. If all the sons of a Judgment have been processed (return from their Convergence node), and the Judgment has not been identified, we can identify it as a Selection structure.

It can be seen from Figure 3 that the identification of while/for structure depends on its Judgment only; and the identification of do-while must depend on the first node (Process or Judgment: node J in A of Figure 3, Judgment can exist in the nesting structure, as shown in Figure 4). The first node in a do-while structure, the Judgment of a while structure and the Convergence of a selection structure are all called key nodes.

\subsubsection{Identification of nesting structure}

According to the execution process of flowchart, the structure first executes to end must be the internal and basic structure. In Figure 4, nesting structures (a) (b) (c) are constructed by the basic structures shown in Figure 3. As each basic structure completes (jump to their Convergence), the out layer structures are executed one by one. So if nesting structures exist, the internal structures must be identified firstly, and then the out layer.

As the identification of a while structure only depend the Judgment node itself (begins and finishes at itself), so if a cycle path appears in the Process node and its current father (comes from) is Judgment, then we can identify the father as a do-while structure. If the Process is the first node (key node) in multi-do-while, we should record the nesting level in the Process node and build a link between the Process node and its current father.

Similarly, if a Judgment node (JN) has been identified as a while/for or selection structure, and a cycle path again appears in the Judgment node, and its current father is Judgment node, then we can identify the father as 


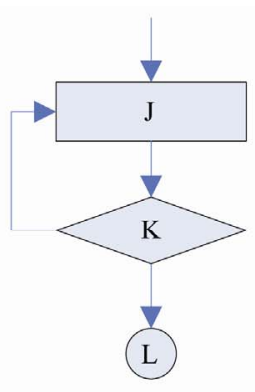

(a) (do -while)

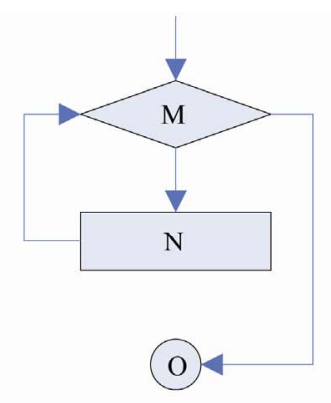

(b) (while/for)

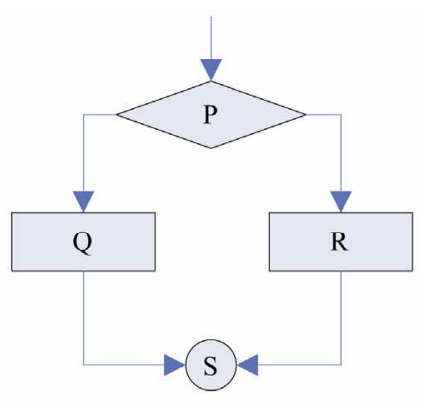

(c) (selection)

Figure 3. Three basic structures.

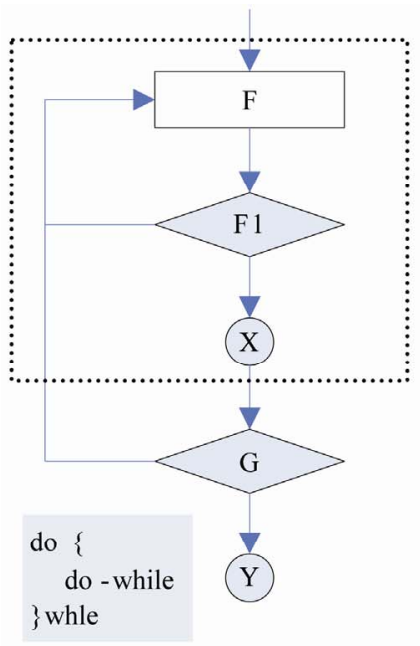

(a) A nested $\mathrm{A}$

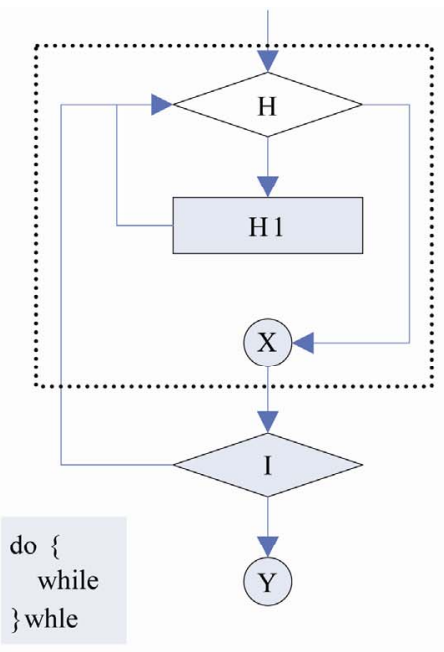

(b) A nested B

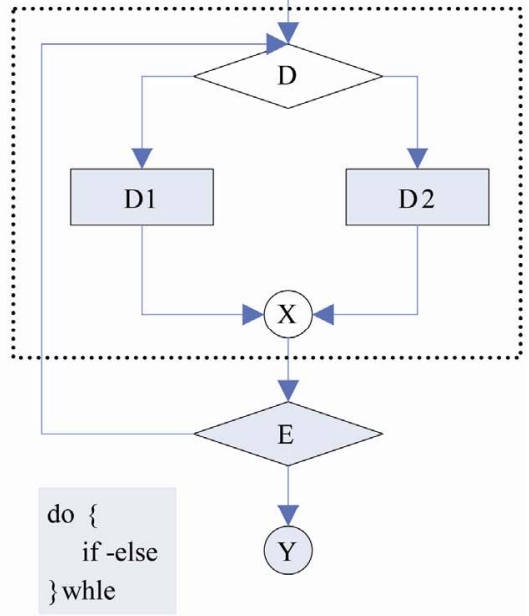

(c) A nested C

Figure 4. Nesting structure of do-while.

a do-while structure. If the Judgment node (JN) is the first node (key node) in a multi-do-while structure, then we should record the nesting level in the Judgment node (JN) and build a link between the Judgment node (JM) and its current father.

As shown in Figure 4, the three figures are all nesting do-while structures. The white nodes in Figure $\mathbf{4}$ are all key nodes. In Figure 4(a) there are two cycle paths in node F, and its current father F1 or G is Judgment, so F1 and $\mathrm{G}$ are both identified as do-while structures; as shown in Figure 4(b), $\mathrm{H}$ is a key node of while structure, meanwhile it is a key node of outer layer do-while structure; as shown in Figure 4(c), D is identified as Selection structure, then a cycle path appears in D, so D is the key node of the outer do-while.

In order to recursively traverse, every Judgment node must be able to have a direct access to its Convergence node, so it can jump current structure to traverse the outer nodes recursively. As a Judgment node and its Convergence is matched, when a Judgment has been traversed, its Convergence must be the subsequent one. So we can use a stack to match them. Define a stack as StackofJudgement, when a Judgment node is first in, we put it into StackofJudgement, when the execution arrive at a Convergence (as currentConvergence), pop the first node (as currentJudgment), and build a link between currentJudgment and currentConvergence, i.e., currentJudgment.Convergence = currentConvergence.

If the basic structures shown in Figure $\mathbf{3}$ are nesting by do-while, we can get the structures shown in Figure 4 While D, F1, H will be identified first, then cycle paths will again appear in F, H, D nodes, so we can know the outer structure must be do-while. Then E, G, I are identified as do-while structure. We should build links between them and G, I, E. Meanwhile the nesting level (as doWhileCounter) of G, I, E should do doWhileCounter++. The program can access G, I, E from D, F, H by the combinative conditions: get the father of $(\mathrm{D}, \mathrm{F}, \mathrm{H})$ and father.doWhileNode $=(D, F, H)$ and father.doWhileCounter $=(D, F, H)$.doWhileCounter 


\subsection{Algorithm Description}

We used a depth-first search algorithm based on recursion. The return conditions of recursion: no need return from sequence; when arrive at a Convergence or End return; when a Judgment has been Identified return, and jump the Convergence of Judgment to process the follow-up nodes.

We process all the sub-nodes recursively when the program arrives at a Judgment. When a Judgment is identified, return to recursive call point.

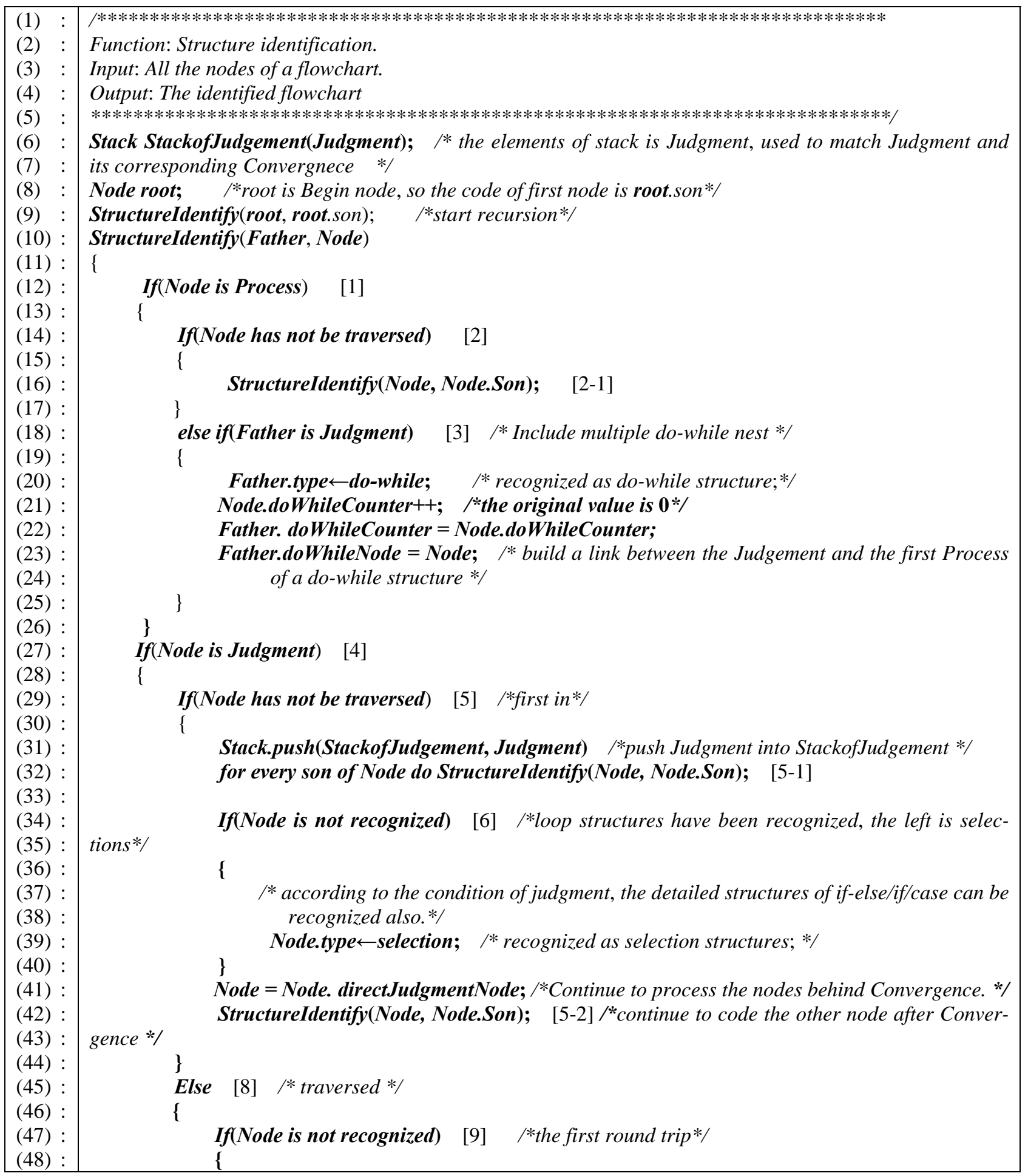




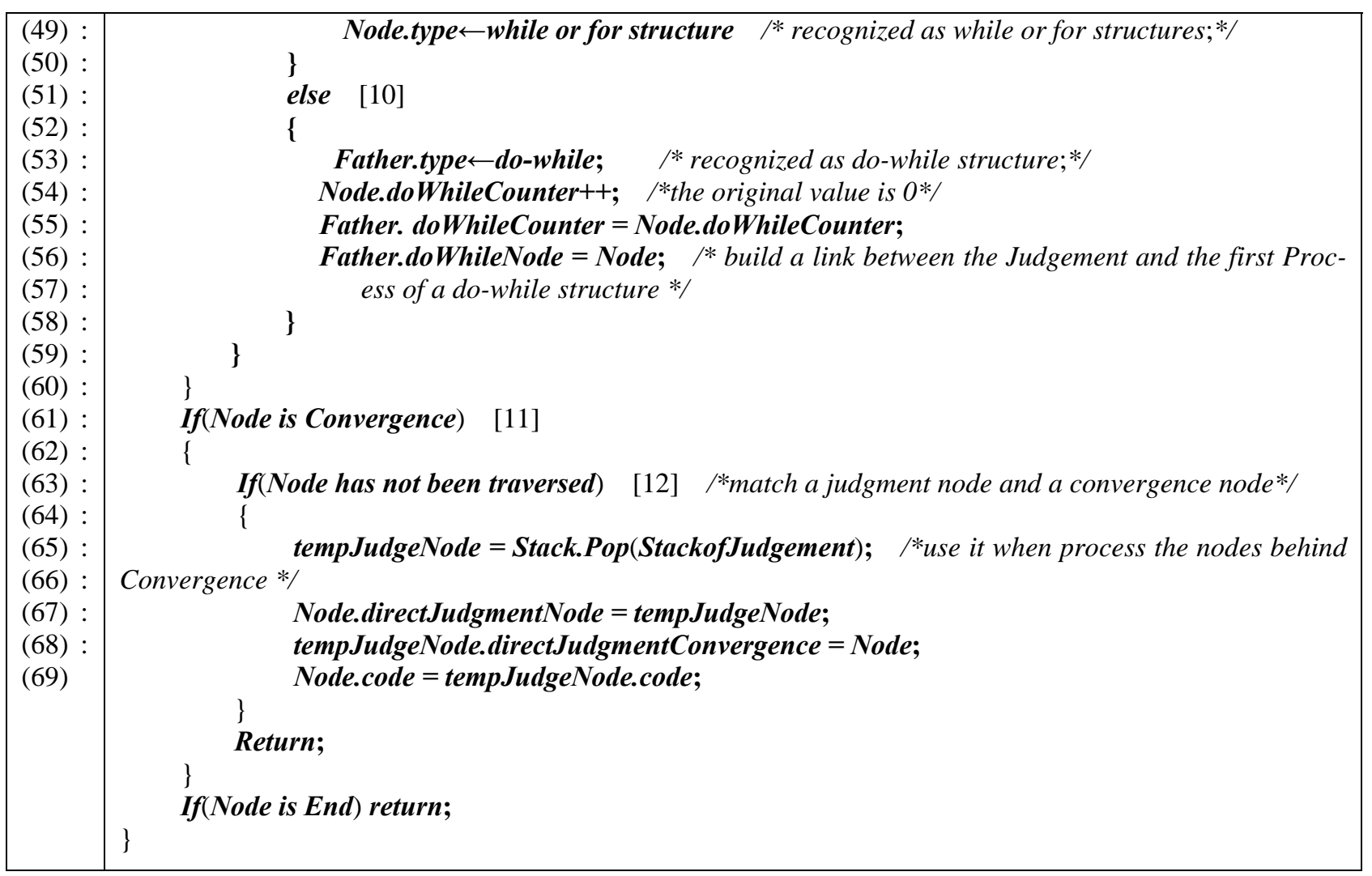

\subsection{Effectiveness Verification of Algorithm}

As the algorithm is based on recursion, so we can use exhaustive method to verify its effectiveness, including the recursive entry and return. For the three basic structures shown in Figures 3, they nest with each other or their own can generate nine nesting structures, as shown in Figures 4-6. We use these twelve structures to verify the effectiveness of the algorithm.

\subsubsection{The Test of Basic Structures}

Take (a) in Figure 3 as a example: Node $\mathrm{J}$ goes into code[2], execute [2-1] (recursion 1); then node $\mathrm{K}$ goes into code [5], execute [5-1] (recursion 2); continue to process node $\mathrm{J}$ or L. 1) Suppose process node J first, J enters code [3], then node $\mathrm{K}$ is recognized as do-while structure and the link between node $\mathrm{J}$ and $\mathrm{K}$ is constructed, return to [5-1] (recursion 2); continue to process node L, enters code [12], construct a link between node $\mathrm{K}$ and L, return to [5-1] (recursion 2); jump code [6], continue to process the other node behind node L. 2) If process $\mathrm{L}$ first, then we can get the same result.

As the process order of Convergence will not affect the result, so in the following discussion we will not discuss it.

Similarly we can check Figure 3(b) and 3(c), also the results are correct.

\subsubsection{The Test of Do-While Nest Structure}

Take (a) in Figure 4 as a example: $F$ goes into [1], then [2], execute [2-1] (recursion 1); F1 goes into [4], [5], and is push into stack, execute [5-1] (recursion 2); continue to process $\mathrm{X}$ or $\mathrm{F}$ (no effect), suppose $\mathrm{F}$ first enters [3], and F1 is identified as do-while structure, build the link between $\mathrm{F}$ and $\mathrm{F} 1$, recursive level(doWhileCounter) of node $\mathrm{F}$ is increased by 1 , then return to [5-1] (recursion 2); node $X$ goes into [12], F1 is popped from stack, construct the link between $F$ and $X$, return to [5-1] (recursion 2), jump [6], execute [5-2] (recursion 3); process node G, $\mathrm{G}$ goes into [5], is pushed into stack, execute [5-1] (recursion 4); Y goes into [12], G is popped from stack, the link between $\mathrm{G}$ and $\mathrm{Y}$ is constructed, return to [5-1] (recursion 4); then $F$ enters into [3], $G$ is identified as do-while structure, construct the link between F and G, recursive level(doWhileCounter) of node $F$ is increased by 1 , return to [5-1] (recursion 4), jump [6], process the successor nodes of node $\mathrm{Y}$.

It can be seen from the above process: First, the basic structure within the dashed box is identified as do-while, then the outer layer.

Similarly we can check Figures 4(b) and 4(c), also the results are correct.

\subsubsection{The Test of While Nest Structure}

Similarly, the inner structure (inside the dashed border) 
was first identified.

Take (a) in Figure 5 as a example: M goes into [5-1], then M1 goes into [5-1], M2 goes into [2], M1 enters [9], $\mathrm{M} 1$ is identified as while structure; jump $\mathrm{X}$ to process $\mathrm{M}$, $M$ enters [9], $M$ is identified as while; jump $Y$ to the successor nodes.

Similarly we can check Figures 5(b) and 5(c), also the results are correct.

\subsubsection{The Test of Selection Nest Structure}

The inner structure (inside the dashed border) was first identified. The outer layer is a single branch selection, that is "if" structure.

Take (a) in Figure 6 as a example: M goes into [5-1], M1 goes into [5-1], then M2 goes into [2], return from X; M3 goes into [2], return from X; continue return to [5-1], enter [6], M1 is identified as selection(if-else). Then jump $\mathrm{X}$, process $\mathrm{Y}$, directly return to the position where $M$ goes into [5-1], then $M$ enters [6], $M$ is identified as selection(if). Jump Y to process the successor nodes.

Similarly we can check Figures 6(b) and 6(c), also the results are correct.

\subsubsection{Summary}

The innermost structure is always identified first, and then the outer layer, each recursive call returns correctly, and all structure identifications are correct. As the above 12 structure covers all nesting structures (continued nesting structure is only a combination of these structures), so we can say the algorithm can correctly identify the structure of structured flowchart.

\section{Code Generation}

\subsection{Algorithm Description}

Take the identified flowchart as input, define a string as output, traverse from root node.

Process: if current node is sequence structure, then

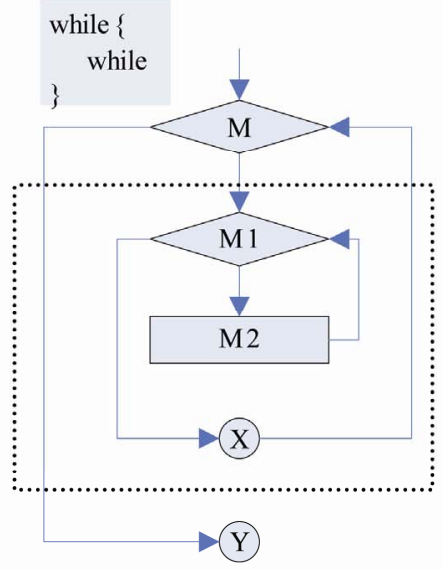

(a) B nested B

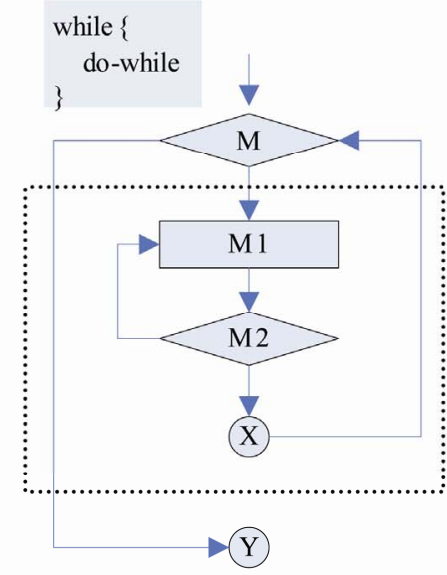

(b) $\mathrm{B}$ nested A

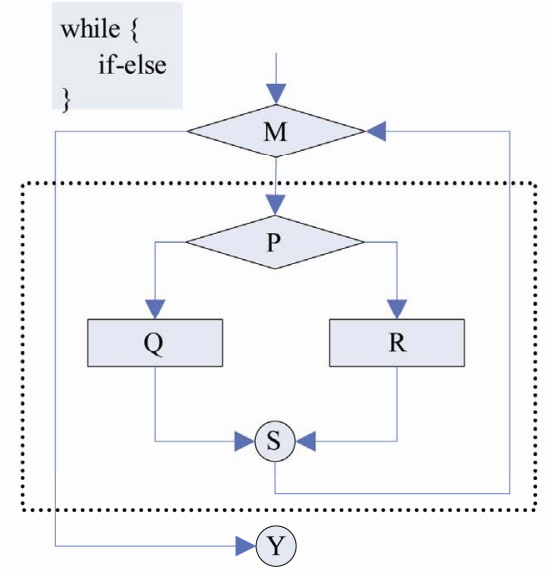

(c) B nested C

Figure 5. Nesting of while.

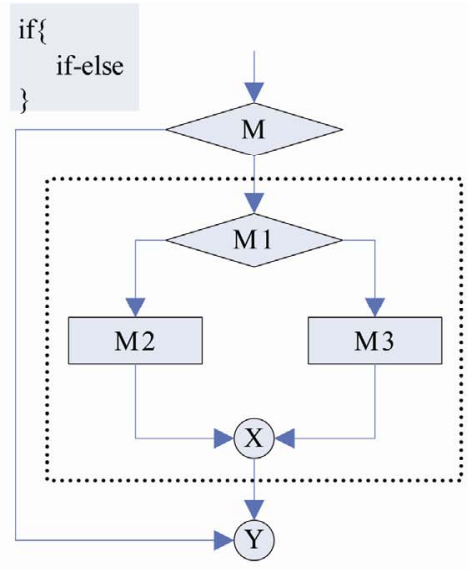

(a) $\mathrm{C}$ nested $\mathrm{C}$

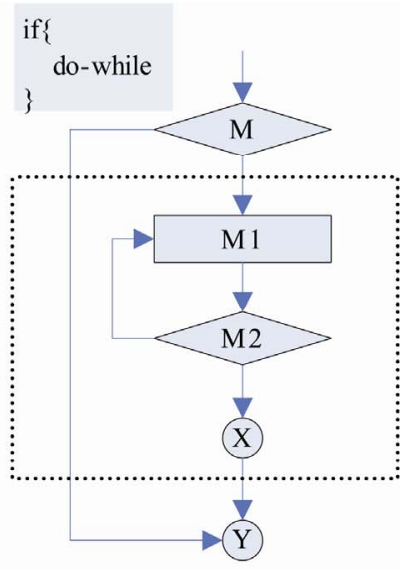

(b) $\mathrm{C}$ nested $\mathrm{A}$

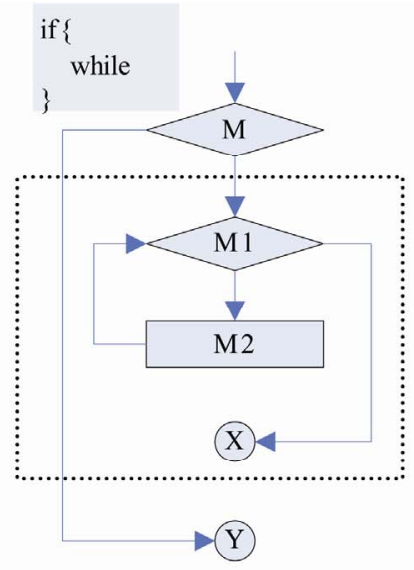

(c) C nested B

Figure 6. Nesting of selection. 
append current code in Process node at output; if current node is selection or loop structures, then define a temp string as tempcode, and process the inside code recursively, append the code in selection or loop at tempcode, when return from recursion, append the tempcode at output.

Return condition of recursion:

1)Selection structure: return when a branch reaches Convergence; if all branches return (all the sons of Judgment have been processed), begin to process suc- cessor nodes of Convergence.

2)Loop structure: a) if Judgment is identified as for/while structure; if it is first in, then process all its sons; if it is not first in, then return and process successor nodes of Judgment. b) if doWhileCounter of Process or Judgment is not equal to 0 , it is shows that current node is the first node of a do-while structure, then process the do-whiles from the outer to inner, when doWhileCounter $==0$, then return from recursion from the inner to outer.

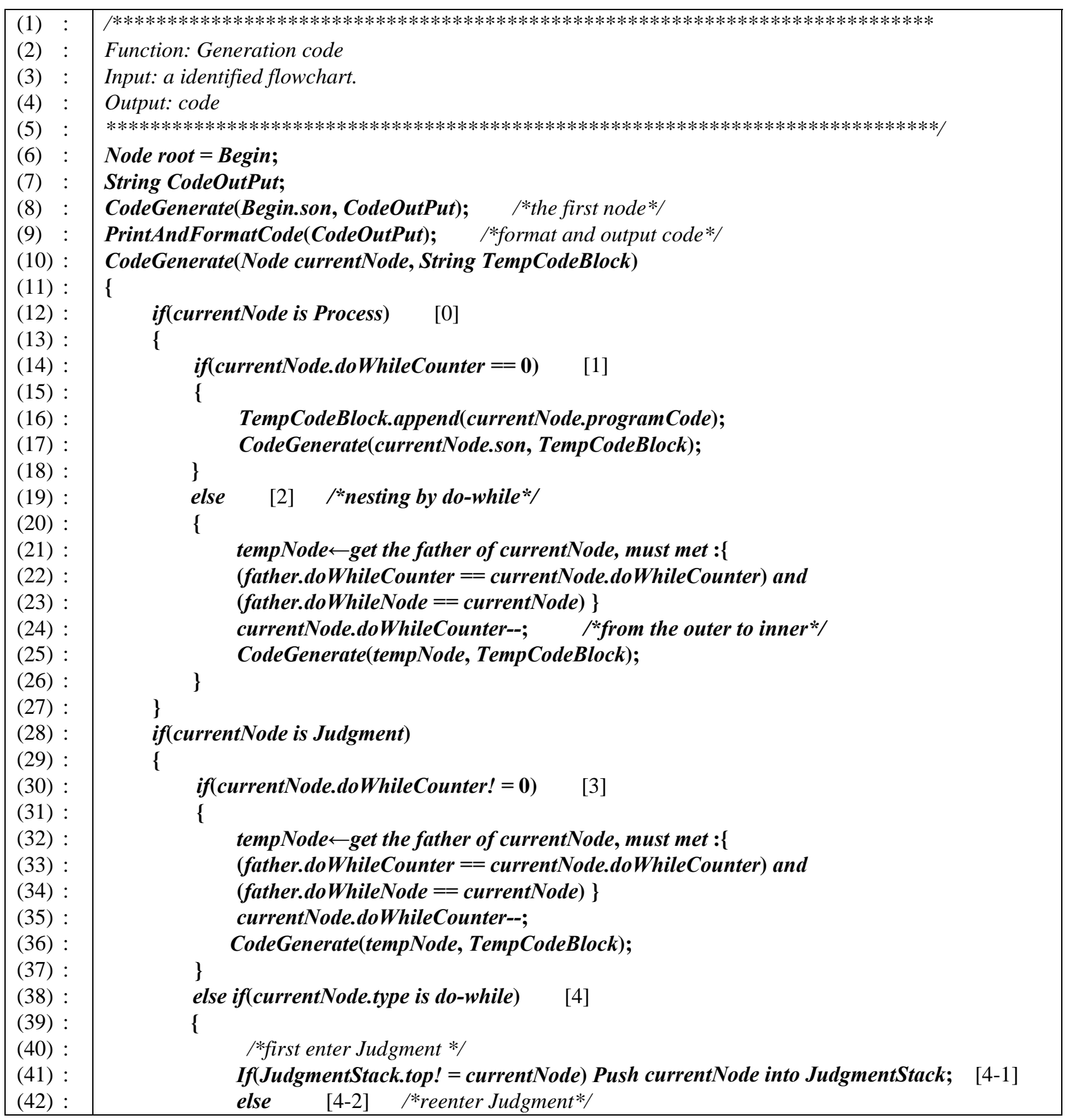




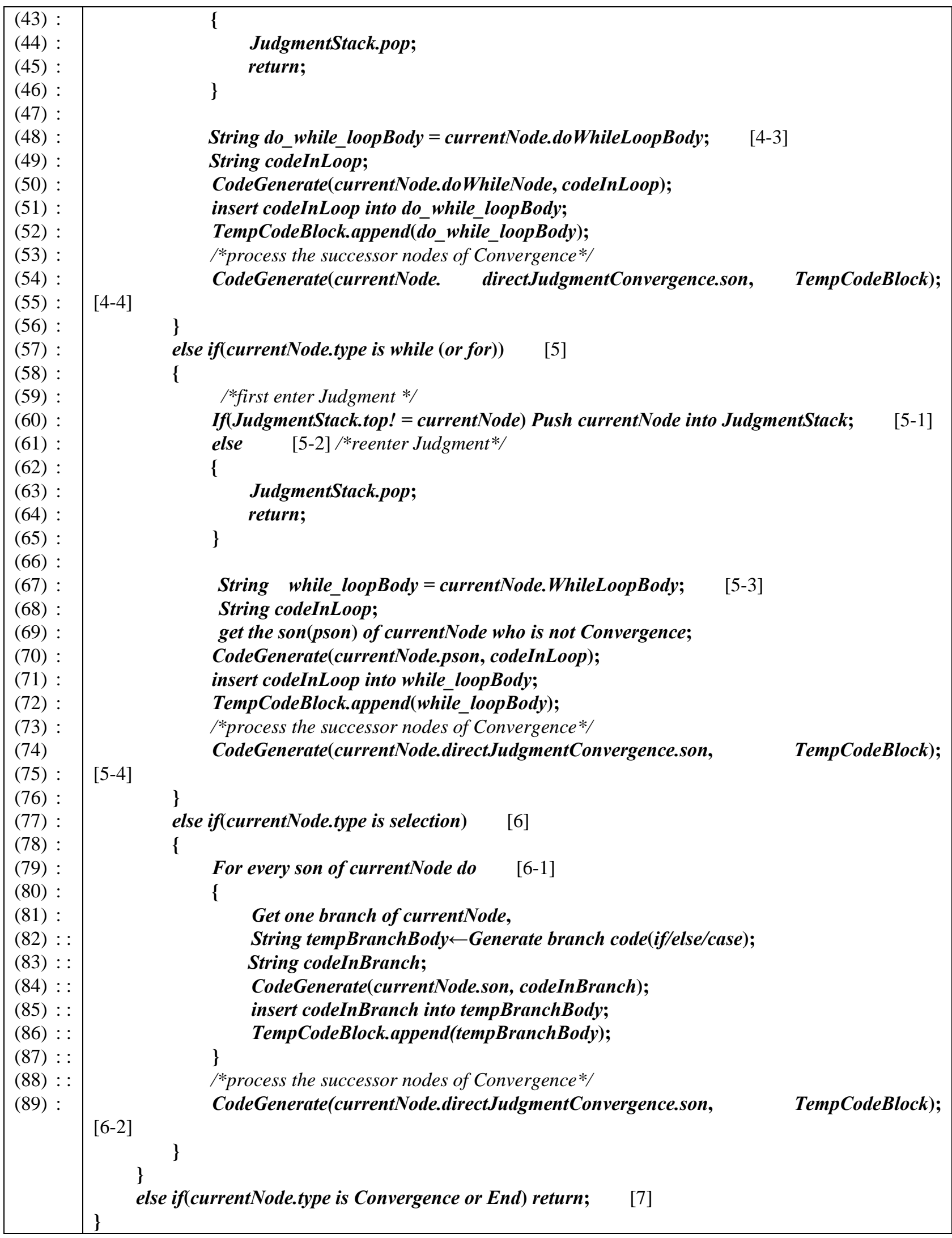




\subsection{Effectiveness Verification of Algorithm}

As the algorithm is based on recursion, so we can use exhaustive method to verify its effectiveness, including the recursive entry and return. We use those twelve structures to verify the effectiveness of the algorithm. The difference from structure identification algorithm is that the identification is from the inner to outer for nest structure, and generation is on the contrary.

\subsubsection{Code Generation for Basic Structure}

Take (a) in Figure 3 as a example: $\mathrm{J}$ goes into [2], $\mathrm{K}$ goes into [4], [4-1], [4-3], then $\mathrm{J}$ enters [1], $\mathrm{K}$ enters [4], [4-2], return to CodeGenerate() of [1]; then return to CodeGenerate() of [4-3]. At this time codeInLoop has been generated, further the entire code of do-while is generated. Execute [4-4], continue to process successor nodes of Convergend.

Similarly we can check Figures 3(b) and 3(c), also the results are correct.

\subsubsection{Code Generation for Nesting Structure}

Take (a) in Figure 4 as a example: $\mathrm{F}$ goes into [2], as $\mathrm{F}$ lies in two do-while structures, so it's doWhileCounter is 2; G goes into [4], [4-1], execute [4-3], generate the outermost do-while framework code; $F$ reenter [2], at this time doWhileCounter==1; then F1 goes into [4], [4-1], execute [4-3], generate the inner do-while framework code; F enter [1], generate the loopbody code of F1; F1 goes into [4], [4-2], return to [4-2] where F1 calls CodeGenerate, generate the inner full do-while code; Then execute [4-4], G goes into [4-2] and return to [4-3] where $\mathrm{G}$ calls CodeGenerate, at this time codeInLoop contains the full code of inner do-while. At last generate the outer complete do-while code, execute [4-4] to process successor nodes.

Similarly we can check the other nesting structures, also the results are correct.

\subsubsection{Summary}

As the above 12 structure covers all nesting structures (continued nesting structure is only a combination of these structures), so we can say the algorithm can correctly generate the code for structured identified flowchart.

\section{Integrated Development Platform}

We developed a integrated develop platform based on Eclipse platform and Graphical Editor Framework (GEF), including flowchart modeling and code automatic generation, to verify the effectiveness of the proposed algorithms.

We construct a model to test various complex nesting structures, including the case of sub-flowchart nest. As shown in Figure 7, there are totally five structures:

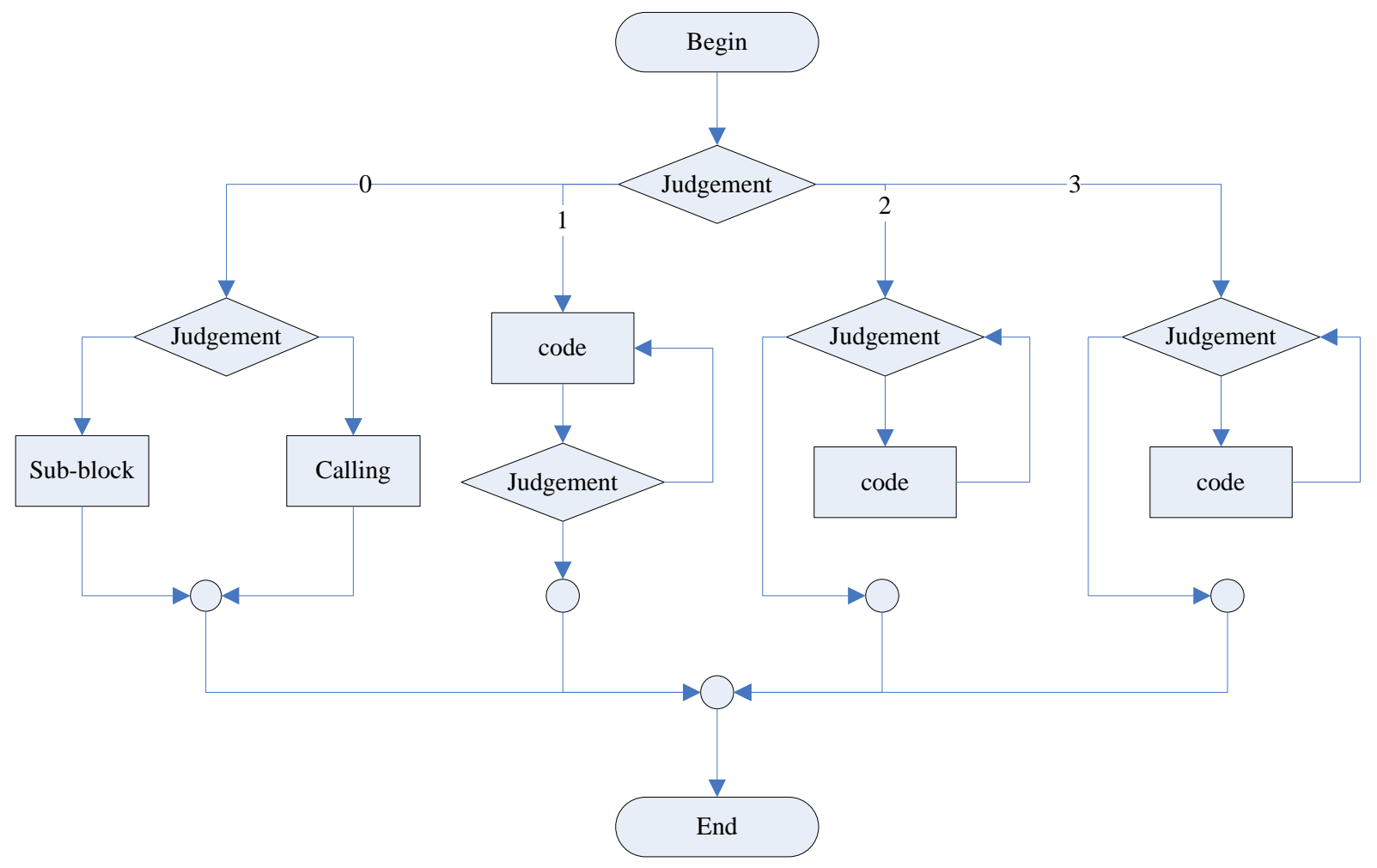

Figure 7. An example of flowchart in integrated development platform. 


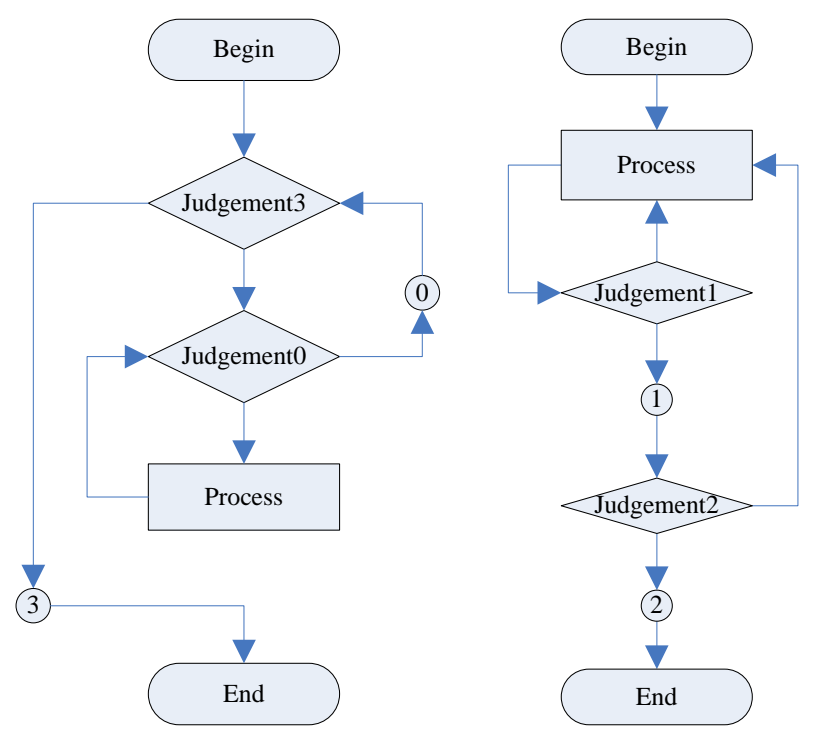

Figure 8. Sub-flowcharts nesting.

switch, for, while, do-while, and if-else. The outer is case, in its first branch we create two sub-flowchart, the type of automatic code generation is source-block (all the code generated will be put in original place) and function-call (all the code generated will be put in fun ction, in original place there will be put a function call statement).

As the program code in TempCode is unformatted, so a third part tools should be called to format that file when they are written into a text file, here we used Codeblocks to do that.

The left figure in Figure 8 is a sub-flowchart of source-block type (while nests while), the right one is function-call (do-while nests do-while).

The code generated automatically is as shown below.

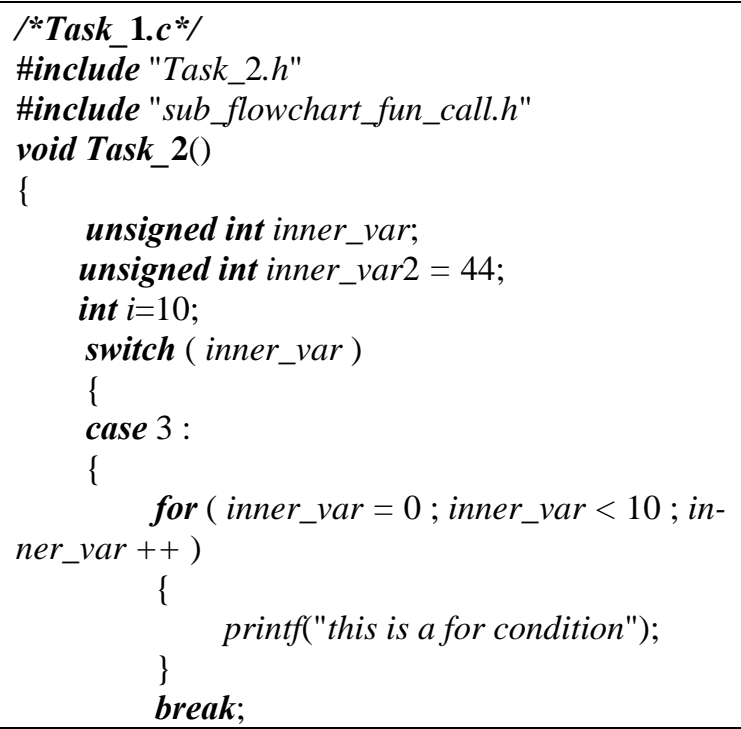

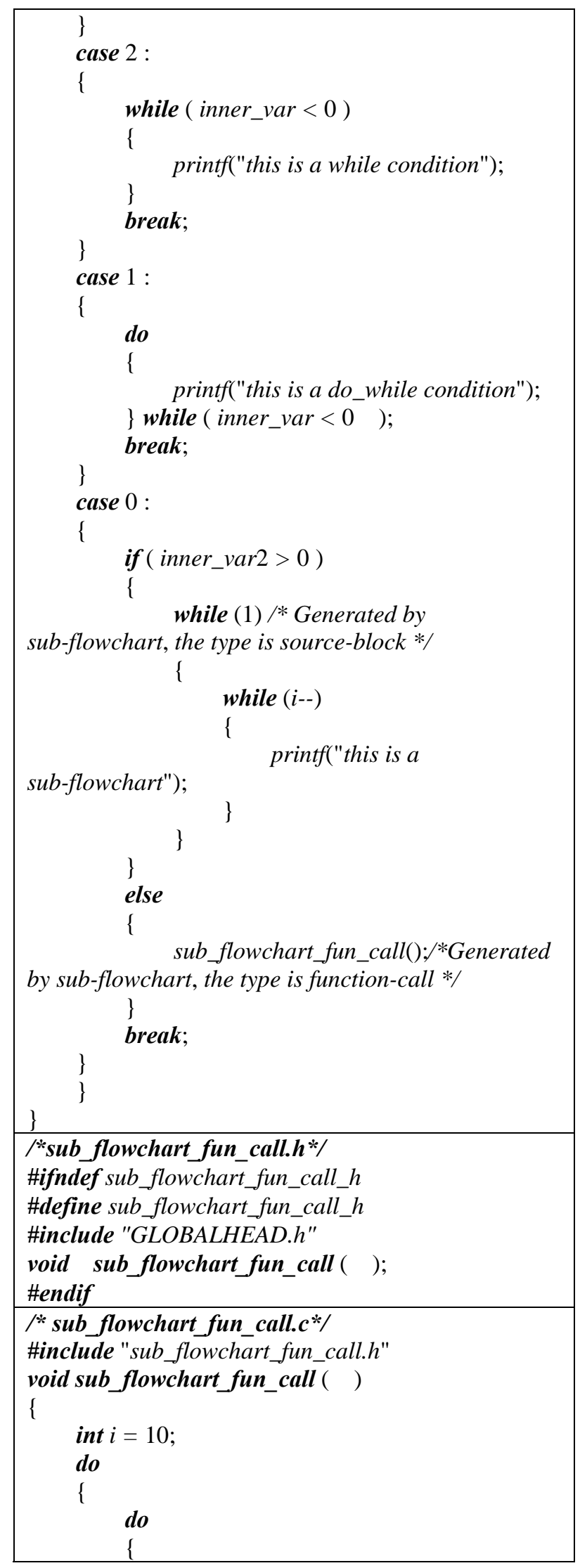




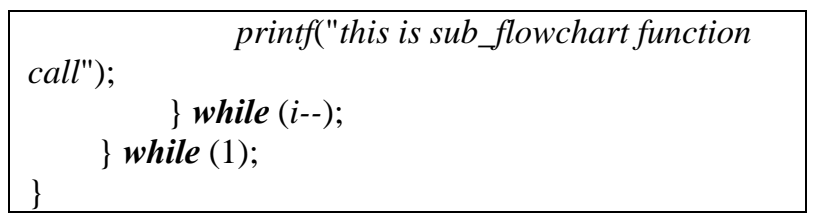

\section{Conclusions}

We proposed a structure identification algorithm for structured flowchart. The effectiveness of the proposed algorithm is checked using exhaustive method, i.e., twelve structures can be identified, then a algorithm can be used to generate code from identified flowchart using recursion algorithm. The technologies and algorithms are used in a integrated development platform, we develop a weapon system based on the platform to verify the effectiveness of the proposed algorithm.

\section{REFERENCES}

[1] T. Zhang, Y. Zhang, X.-F. Yu, et al., "MDA Based Design Patterns Modeling and Model Transformation," Journal of Software, Vol. 19, No. 9, 2008, pp. 2203-2217.

[2] R.-F. Lv, G. Wang, X.-X. Wen, et al., "Process Modeling Method of Calculation Independent Model Level Based on MDA," Computer Integrated Manufacturing Systems, Vol. 14, No. 5, 2008, pp. 868-874.

[3] S. Needham, "OMG Unified Modeling Language Specification,” Object Management Group, 2003, pp. 275-293. http://www.digilife.be/quickreferences/Books/OMG\%20 UML\%20Specification\%201.4.pdf

[4] Z. K. Zhao, Q. J. Sheng and Z. Z. Shi, “An Execution Semantics of UML Activity View for Workflow Modeling," Journal of Computer Research and Development, Vol.42, No. 2, 2005, pp. 300-307.

[5] R. Eshuis and R. Wieringal, "A Formal Semantics for UML Activity Diagrams,” Tech Report, University of Twente, 2001, pp. 201-204.

[6] H. Jiang, D. Lin and X. R. Xie, “The Formal Semantics of
UML State Machine,” Journal of Software, Vol. 13, No. 12, 2002, pp. 2244-2250.

[7] D. Harel, "Statecharts: A Visual Formalism for Complex Systems," Science of Computer Programming, 2007.

[8] J.-L. Shen, L.-Z. Wang, X.-D. Li, et al., “An Approach to Generate Scenario Test Cases Based on UML Sequence Diagrams," Computer Science, Vol. 31, No. 8, 2004, pp. 1-6.

[9] S. Raman, N. Sivashankar and W. Stuart, "HIL Simulators for Powertrain Control System Software Development," American Controls Conference, St. Louis, Missouri, USA, 2009, pp. 23-32.

[10] S. Sendall and W. Kozaczynski, "Model Transformation: The Heart and Soul of Model-Driven Software Development," IEEE Software, Vol. 9, 2003, pp. 42-45. doi:10.1109/MS.2003.1231150

[11] H. Dakhore and A. Mahajan, "Generation of C-Code Using XML Parser,” http://www.rimtengg.com/iscet/proceedings/pdfs/advcom p/149.pdf

[12] M. C. Carlisle, T. A. Wilson, J. W. Humphries, et al., "Raptor: Introducing Programming to Non-majors with Flowcharts,” Journal of Computing Sciences in Colleges, Vol. 19, No. 4, 2004, pp. 1-6.

[13] T. Watts, “The SFC Editor: A Graphical Tool for Algorithm Development," Journal of Computing in Small Colleges, Vol. 20, No. 2, 2004, pp. 73-85.

[14] K. Charntaweekhun and S. Wangsiripitak, "Visual Programming Using Flowchart," International Symposium on Communications and Information Technologies, ISCIT '06, 20 September - 18 October 2006, pp. 1062-1065.

[15] I. Nassi and B. Shneiderman, "Flowchart Techniques for Structured Programming," ACM SIGPLAN Notices, Vol. 8, No. 8, 1973, pp. 12-26. doi:10.1145/953349.953350

[16] J. F. Gimpel, "Contour: A Method of Preparing Structured Flowcharts,” ACM SIGPLAN Notices, Vol. 15, No. 10, 1980, pp. 35-41. 